\title{
Equilibrium assignment model of road network for traffic flow in open pit
}

\author{
Xiao-yu Sun ${ }^{1, a}$, Song-song Zhao ${ }^{2, b^{*}}$, Heng Liu ${ }^{3, c}$, Hang Zhang ${ }^{4, d}$ \\ School of Resources and Civil Engineering, Northeastern University, Shenyang 110004, China \\ àsunxiaoyu@mail.neu.edu.cn, ${ }^{\text {b }} 1719219539 @ q q . c o m,{ }^{c} 215761002 @ q q . c o m, \quad{ }^{d}$ huhuhang@gmail. \\ com
}

Keyword: truck dispatching; wardrop principle; comprehensive resistance; equilibrium distribution.

\begin{abstract}
In order to further improve the truck dispatching theory of open pit mine, an open pit mine truck dispatching equilibrium distribution model based on traffic flow planning was put forward. The distribution model is based on the principle of Wardrop, and the distribution of the road resistance is used as the basis of the distribution. Verified by the case, the road traffic flow of the model in the truck dispatching process is more optimized as the research object than the result of traffic planning , and achieves the purpose of further optimization of truck dispatching process.
\end{abstract}

\section{Introduction}

As an important part of the open pit mine production, the truck dispatching process has always been the key research direction of the optimization of the open pit mine ${ }^{[1,2]}$. At present, the main research methods divide the open pit mine truck scheduling process into three stages respectively: the shortest path optimization, traffic planning and real-time scheduling ${ }^{[3,4]}$. But in the calculation of the shortest path and traffic flow optimization, trucks on the road travel time only as a linear function of the path were considered and the impact of the link travel time of other vehicles on the road and road traffic ability of wagon flow allocation results did not have related literature research.But in the field of traffic, a large number of research results shows that the travel time of the car on the links and road traffic flow are closely related, and road traffic capacity has a obvious limit to road traffic distribution ${ }^{[5,6]}$.

To direct at the above problems, this paper firstly analyzes the accuracy of the traditional traffic planning of the maximum production under the condition of continuous changed truck's quantity.Then, Through establishes the equilibrium assignment model of open-pit mine truck dispatching based on the traffic planning studying the truck traffic flow of open pit mine under the comprehensive resistance, and tests the effects of comprehensive resistance on traffic distribution and the practicability and reliability of the equilibrium assignment model with a case, provides a theoretical basis and guidance for a more comprehensive optimization of truck dispatching process.

\section{Equilibrium assignment model}

To optimize the open-pit truck dispatching process, some assumptions were made in [7] refs. Open-pit mine equilibrium assignment model firstly by maximum yield as the initial target of traffic programming model to give the trucks distribution amount, then establish network traffic equilibrium assignment of traffic assignment.

The goal of traffic planning of maximum production in the open pit mine is:

$$
\max Z=W \sum_{i}^{N} \sum_{j}^{M} q_{i j}
$$

Traffic planning needs to satisfy some constraints of the existing production conditions of mine, the limit of production capacity of loading point is: 
$\sum_{j=1}^{N} q^{(i, j)} \leq \frac{8 * 60}{t_{z}}$

The limit of ore reserves of the loading point is:

$W \sum_{j=1}^{N} q^{(i, j)} \leq R_{\text {ore }}^{i}$

The limit of block reserves of the loading point is:

$W \sum_{j=1}^{N} q^{(i, j)} \leq R_{\text {rock }}^{i}$

The limit of production capacity of unloading point is:

$$
\sum_{i=1}^{M} q^{(i, j)} \leq \frac{8 * 60}{t_{x}}
$$

The limit of output of unloading point is:

$$
\sum_{i=1}^{M} q^{(i, j)} \geq B_{j}
$$

Calculate the maximum number of transport with the last truck in each path, the limit of the number of truck is:

$$
\sum_{i=1}^{M} \sum_{j=1}^{N} x_{i j} /\left(480-\left(\left(t_{i j} / p\right)-1\right) p / t_{i j}\right) \leq U
$$

The limit of grade of unloading point is:

$$
\alpha \leq \frac{W^{*} \sum_{i=1}^{M} q^{(i, j)} * \theta_{i}}{W^{*} \sum_{i=1}^{M} q^{(i, j)}} \leq \beta
$$
follows:

The equilibrium assignment model for traffic flow of open pit based on traffic planning is as

$$
\begin{array}{ll}
\min & \sum_{a} \int_{0}^{x_{a}} t_{a}(x) d x \\
\text { s.t. } & \sum_{a} \int_{0}^{x_{a}} f_{k}^{(i, j)}=q^{(i, j)} \\
& f_{k}^{(i, j)} \geq 0, \forall k \in K^{(i, j)},(i, j) \in K \\
& x_{a}=\sum_{w} \sum_{k} f_{k}^{(i, j)} \delta_{a, k}^{(i, j)}, \forall a \in A \\
& x_{a} \geq 0, t_{a} \geq 0, f_{k}^{(i, j)} \geq 0, q^{(i, j)} \geq 0
\end{array}
$$

This paper choose widely used function model of $\mathrm{BRP}^{[8,9]}$ in the field of traffic as the 
rcomprehensive resistance of mine carrying. Resistance model of BRP is obtained from the United States Highway Bureau by the investigation of a large number of road data and multiple practice, the specific formula is as follows:

$$
t_{a}=t_{0}\left(1+\alpha\left(\frac{X_{a}}{C}\right)^{\beta}\right)
$$

The initial traffic distribution $q^{(i, j)}$ between the loading and unloading points, is given by the traffic planning model in the last section. Formula (11) is the conservation relation between the path flow and the traffic distribution; the formula (12) is the relationship between the path flow and the link flow; the formula (13) is a non -negative constraint.

Where A: set of links of road network; $i 、 j$ : loading and unloading point in road network; $m, N$ : total number of loading and unloading point in road network; $t_{z}$ : shovel's loading time at loading point; $t_{x}$ : truck's unloading time at unloading point; $R_{\text {ore: }}^{i}$ ore quantity of loading point $i$; $R_{\text {rock: }}^{i}$ rock quantity of loading point; $O_{j}$ : planning quantity of unloading point; $\alpha, \beta, \theta$ : the upper and lower and actual grade of ore at unloading point; $W$ : trucks carrying capacity; $U$ : total number of trucks; $f_{k}^{(i, j)}$ : the flow of path $k$ of pair $(i, j)$ between loading and unloading point; $c_{k}^{(i, j)}$ : resistance of path $k$ of pair $(i, j)$ between loading and unloading point, where sum of related links; $c^{(i, j)}$ : resistance of the shorter path of pair $(i, j)$ between loading and unloading point; $q^{(i, j)}$ : truck distribution of pair $(i, j)$ between loading and unloading point, where carrying number of truck; $K^{(i, j)}$ : sets of path of pair $(i, j)$ between loading and unloading point; $K$ : set of pair $(i, j)$ between loading and unloading point; $x_{a}$ : flow of link $a$; $t_{a}\left(x_{a}\right)$ : resistance function of link $a ; \delta_{a, k}^{(i, j)}$ : related variables of connection between links and path; when link $a$ belong path $k, \delta_{a, k}^{(i, j)}=1$, or $\delta_{a, k}^{(i, j)}=0$. $\mathrm{t}_{a}$ is travelling time on link $a$; $\mathrm{t}_{0}$ is free flow time on link; $x_{a}$ is traffic flow on link $a$ where is truck volume; $C$ is the traffic capacity of link; $\alpha, \beta$ is a constant value as 0.15 and 4 respectively ${ }^{[10]}$.

\section{Analysis of case}

In order to test the practicability and rationality of the model, the production process of open pit in the first section was used to verify the model.

An open pit mine use the production technology of truck-excavator has 10 loading points and 8 unloading points, whicn 1 5 are spots of unloading ore and $6 \sim 8$ are spots of unloading block. This mine totally has 10 excavators, 68 sets trucks of 154 ton of load weight, the average time of shovel loading is $3 \mathrm{~min}$, unloading time at unloading point is $1.5 \mathrm{~min}$, the average speed is $30 \mathrm{~km}$.

The traffic capacity of links in mine refer to the mining manual ${ }^{[11]}$ as table 1 . In this case, the traffic capacity of the arterial line on the level 1 is 100/h, level 2 is 50/h and the branch line is $25 / \mathrm{h}$.

Table 1 traffic capacity of links

\begin{tabular}{ccc}
\hline Grade of road & Annual freight volume/[kt] & Traffic capacity/[veh/h] \\
\hline Level 1 & $>12000$ & $>85$ \\
Level 2 & $3500 \sim 12000$ & $25 \sim 85$ \\
Level 3 & $<2500$ & $<25$ \\
\hline
\end{tabular}

In a shift, each unloading point requirements in accordance with the mine production plan such as table 2 shows. The ore reserves / rock reserves and the average grade of each loading point are shown in table 3 , grade requirements of unloading ore point is $32 \%+0.5 \%$.

Table 2 Yield of unloading point[ten thousand ton]

\begin{tabular}{ccccccccc}
\hline Unloading ponit & 1 & 2 & 3 & 4 & 5 & 6 & 7 & 8 \\
\hline Production & 2.60 & 2.48 & 2.20 & 2.98 & 2.40 & 4.20 & 3.80 & 3.65 \\
\hline
\end{tabular}


Table 3 Ore reserves / rock reserves and grade loading point[ten thousand ton]

\begin{tabular}{cccc}
\hline Loading ponit & Ore reserves & Rock reserves & Grade \\
\hline 1 & 2.24 & 2.68 & 33.5 \\
2 & 2.80 & 3.36 & 31.2 \\
3 & 2.65 & 3.18 & 32.5 \\
4 & 2.05 & 2.45 & 30.8 \\
5 & 2.74 & 2.38 & 33.4 \\
6 & 2.50 & 3.00 & 34.2 \\
7 & 2.46 & 2.96 & 35.0 \\
8 & 1.98 & 2.36 & 32.7 \\
9 & 2.28 & 2.74 & 30.2 \\
10 & 2.32 & 2.78 & 32.5 \\
\hline
\end{tabular}

\section{Result and analysis of traffic flow under two different states}

Comparison of traffic flow under two different states. In order to analyze the traffic planning section of the truck allocation results better, draw the ratio of traffic flow and the capacity of each link of road network with using TransCAD as a display. Convert truck traffic flow of links to same units consistent with the flow of hours of road capacity, and then the traffic flow in the road network is shown in the road network as shown in figure 1 . For make a result comparison, make the distribution results under linear resistance called distribution of assignment of free flow and the distribution results based comprehensive resistance called assignment of equilibrium flow, the result of equilibrium flow is shown in Figure 2.

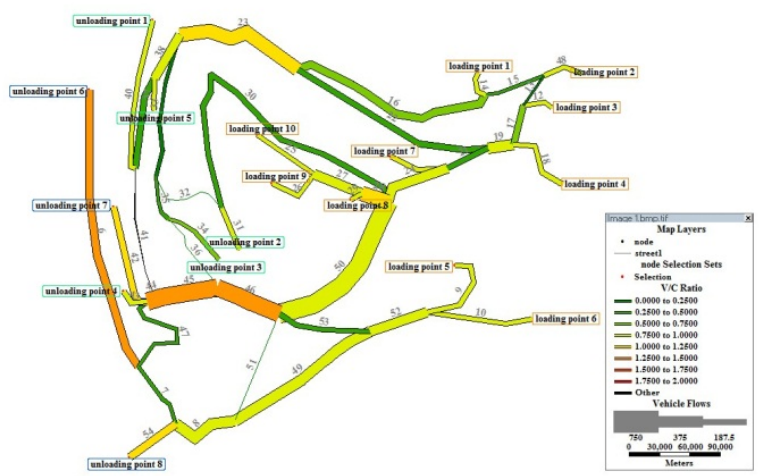

Fig1 Truck distribution of traffic planning

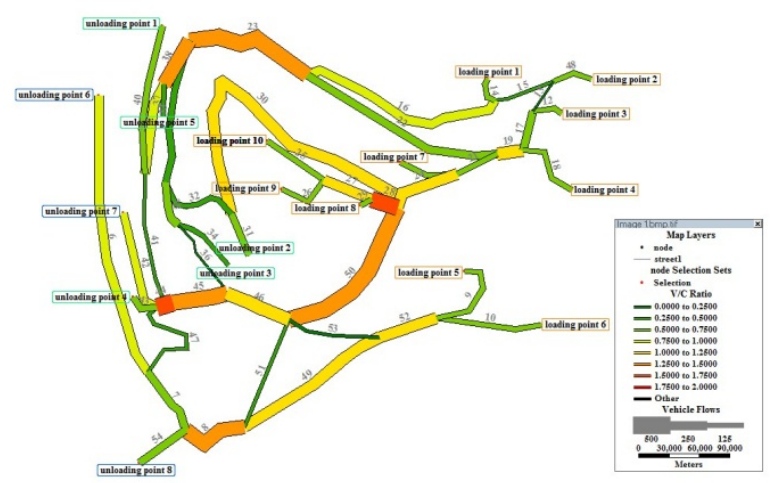

Fig.2 Truck traffic distribution of links of equilibrium flow

The width of links in figures means the size of the traffic flow, the larger the road traffic, the wider the flow. The color of links represent the sieze of the ratio between traffic flow and traffic capacity that is the numerical size of $\mathrm{V} / \mathrm{C}$, and the bigger the value, the deeper the color. When the ratio beyond 1 , the link occurs congestion. As shown in the figure1,the traffic flow of the links in main line of second leve such as No.2, 23, 44, 45, 46 and the regional line such as No.6、 54 beyond its traffic capacity apparently that will occur congestion, No.6 occurs congestion especially, which seriously restrict the unloading point loading and unloading efficiency, and converse with the assumption that the flow of traffic planning is free. Therefore, there is a certain blindness in the traffic planning to allocate the truck in the open pit mine.

Compared with the distribution results of traffic planning flow and equilibrium flow shown in figure 2, links of larger value of ratio between traffic flow and traffic capacity increases significantly, but increased links mainly belong to arterial line of level 1 and level 2, such as link 8, 23, 28 and 50. All links flow belong to the branch line of loading and unloading point below traffic capacity of links, and it is significantly improved of the low efficiency of loading and unloading point caused by the congestion of branch line under the free flow . This distribution is consistent 
with the traffic congestion in the road network, which shows the rationality of the results. At the same time, the results show that the method can greatly improve the distribution of traffic flow and improve the efficiency of loading and unloading points.

Comparison of parameters of traffic flow under two different states. For making a detailed comparison of main parameters like amout of traffic flow and the travelling time of every link , using Matlab draw a graphics to analyze the result with the line chart as shown in Figure 3, 4.

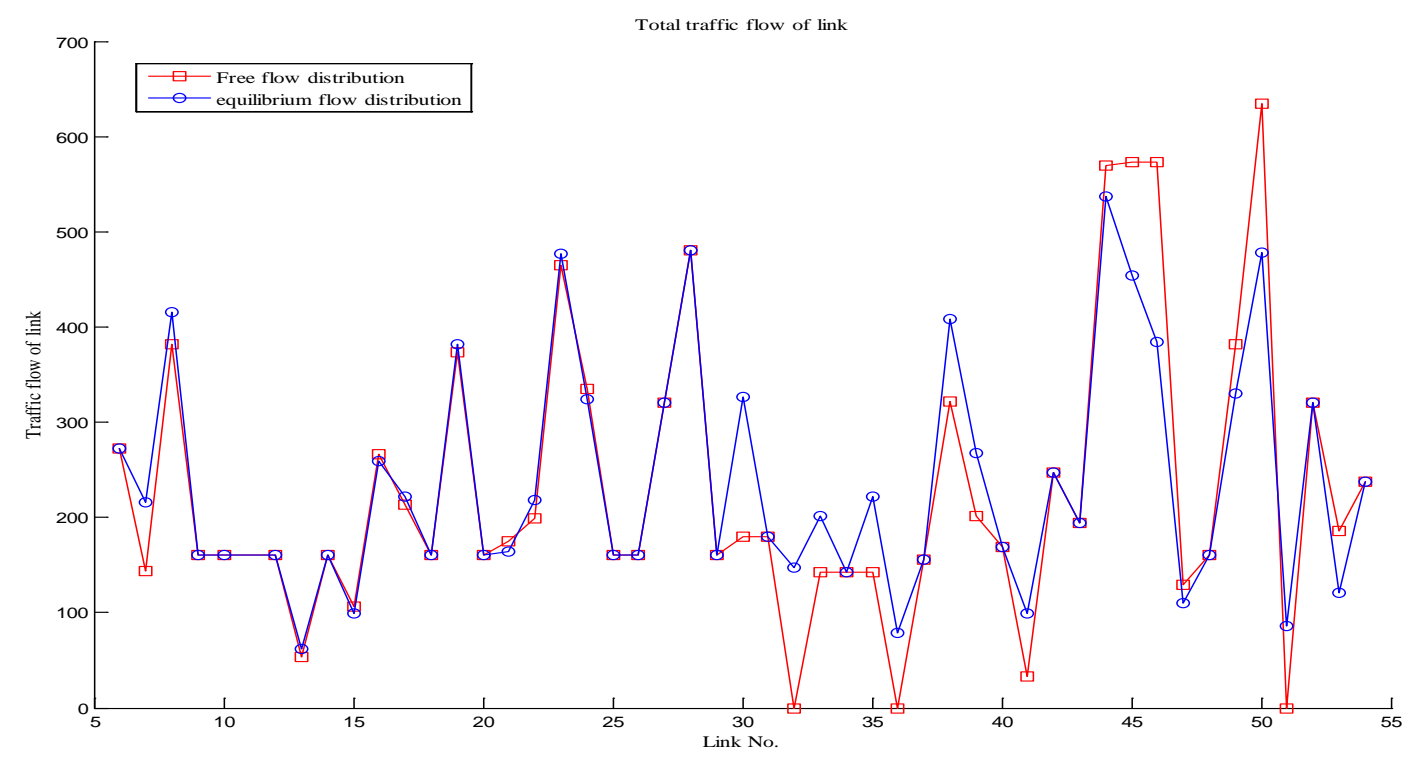

Fig.3 Total traffic flow of link

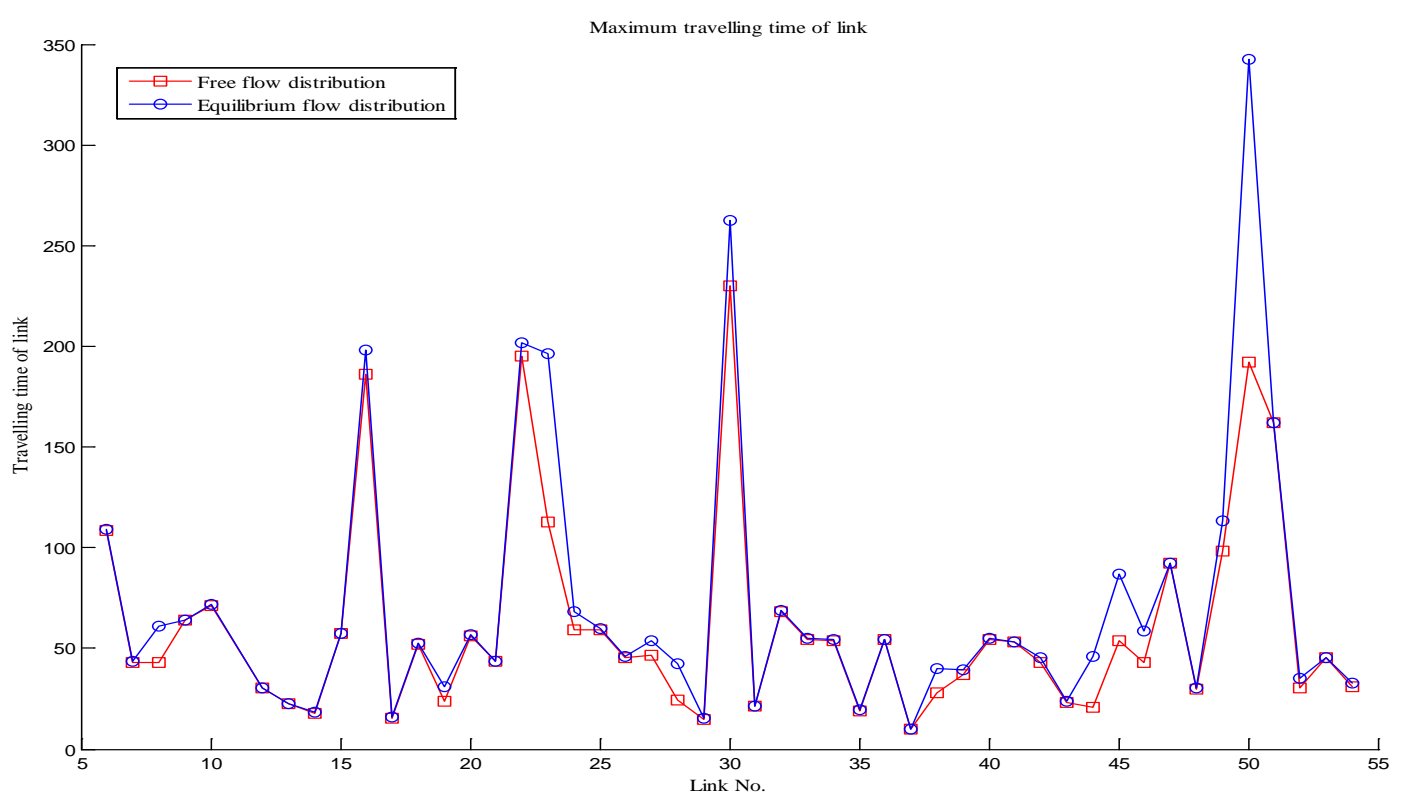

Fig.4 Maximum travelling time of link

The total traffic flow and maximum travelling time of link under the condition of traffic planning and equilibrium flow are analyzed. When the value of traffic flow beyond the traffic capacity of link, truck will happen to congest and the travelling time also increase significantly, which is consistent with the situation of actual production. The travelling time of $t$ is same basically before link 45 of two kind of distribution, it can be seen there is little or no traffic flow before link 45 . But they are not keep consistent after link 45 because there is large traffic flow on the link and 
these travelling time are not a state of free flow under the condition with less traffic flow.

The total traffic flow on link of two kinds of distribution remain basically the same before link 30 seen from the Fig 3, and the value of equilibrium flow is larger than the value of traffic planning low after link 30, but all the maximum value of traffic flow of equilibrium distribution is smaller than the traffic planning distribution. All these analysis show that the equilibrium distribution can balance traffic flow, which makes travelling time of each link trend to a more equilibrium state.

\section{Summary}

The traffic planning model of the existing theory of truck dispatching don't consider the limit of traffic capacity of link in the optimization process, its shortest path choosen by the linear resistance based on the length of link. The result of distribution does not comply with assumption that the traffic flow is free on each link so that the optimization result is not reasonable.

Based on the model of traffic planning model and considering the comprehensive resistance whicn have an impact on travelling time of link influenced by traffic flow and traffic capacity, the equilibrium assignment model can get more optimized distributed result of traffic flow. The distribution of each link can make the travelling time of truck shortest, and is more reasonable and better compared with traffic planning model, offers a theoretical guidance for the optimization of truck dispatching used in open-pit mine, provides a scientific basis for implementing the model on actual production of mine.

\section{Reference}

[1] Bastos G S. Decision Making applied to Shift Change in Stochastic Open-pit Mining Truck Dispatching[J]. IFAC Proceedings Volumes, 2013, 46(16): 34-39.

[2] Zhang L, Xia X. An Integer Programming Approach for Truck-Shovel Dispatching Problem in Open-Pit Mines[J]. Energy Procedia, 2015, 75: 1779-1784.

[3] Su Jing, Zhang Youti. Study on truck scheduling theory of open pit mine[J]. Journal of China Coal Society, 1997, 22(1): 52-55.

[4] Gurgur C. Z.,Dagdelen, K., Artittong, S.. Optimisation of a real-time multi-period truck dispatching system in mining operations[J]. International Journal of Applied Decision Sciences, 2011, 4(1): 57-79.

[5] Hou B, Li X. User equilibrium with length constrained users[C]//Computational Sciences and Optimization (CSO), 2012 Fifth International Joint Conference on. IEEE, 2012: 494-499.

[6] Wen Xuli, Wu Wen, Cao Rui. Characteristic comparison and parameter selection of several typical road resistance function[J]. Journal of Highway and Transportation Research and Development(Application Technology), 2014, (2): 205-208.

[7] Zhang Yingjie. Application of fuzzy optimization in vehicle scheduling of open pit mine[D]. Dalian: Dalian Maritime University, 2008.

[8] Chen J, Mei Z, Wang W. Road resistance model under mixed traffic flow conditions with curb parking[J]. China Civil Engineering Journal, 2007, 40(9): 95-100.

[9] Wu Xiaoceng, Fan Binquan. Equilibrium problems for dynamic traffic networks with additional path flow constraints[J] .Chinese Journal of Engineering Mathematics , 2006 , 23(4): 607-613.

[10] Du L, Han L, Chen S. Coordinated online in-vehicle routing balancing user optimality and system optimality through information perturbation[J]. Transportation Research Part B: Methodological, 2015, 79: 121-133.

[11]Wang Yunmin, Modern Mining Handbook[M]. Beijing: Metallurgical industry press, 2012. 